\title{
A NEW ENERGY PATTERN FACTOR METHOD TO ESTIMATE WEIBULL SHAPE PARAMETER FOR IMPACT RESISTANCE OF CONCRETE
}

\section{PARSHYAM NAGAVENI, PALLEM BHARGAVI, VINEESHA REDDY GOSPATI \& G. MURALI}

\author{
School of Civil Engineering, SASTRA Deemed to be University, Thanjavur, India
}

\begin{abstract}
Impact resistance is an essential consideration in the design of civilian and military infrastructure, it should be designed precisely. In recent times, the distribution of Weibull has often been used, acknowledged, and suggested distribution in published works for many concrete applications. This work proposes a new approach for estimating the distribution of the Weibull shape parameter for impact resistance of concrete for the machine foundations. This new approach is called the energy pattern factor method. The experimental impact test data were collected from the earlier literature, which involves 1174 values, and these data were analysed using two-parameter distributions of Weibull. The Fittingness of the proposed equation is verified based on four levels of validation approach. This new approach was established and the final three equations (Polyratio (3,3), Logistic, and DR-Hill) were cleared the four level validation which indicates the good accuracy.
\end{abstract}

KEYWORDS: Weibull, Shape Parameter, Impact, Energy Pattern Factor \& Validation

Received: Jun 06, 2020; Accepted: Jun 26, 2020; Published: Jul 28, 2020; Paper Id.: IJMPERDJUN2020499

\section{INTRODUCTION}

Due to the more numerous acts of terrorism worldwide, there has been widespread attention in civil and military infrastructure development to improve their impact resistance. However, a widely acknowledged that the concrete behaves brittle, the degree of brittleness increased with increased compressive strength. Fibre addition in concrete counteracts its brittleness, increasing the impact strength up to fifteen times [1,2], predominantly delays the development of crack formation. The expansion and the growth of cracks in concrete make it unable to overwhelmed the internal stress. The fibres themselves bear some of these internal stresses.In contrast, the excessive stresses are imparted to the vicinity in the matrix, and this behavior attributes to the fibrous concrete being remarkable over the non-fibrous concrete. As the impact resistance offered by plain concrete is wholly inadequate, the crack develops randomly and propagates in the structures. Hence, the durability and mechanical properties of concrete become diminished, and therefore the material cannot deliver the performance expected. Fibre addition has the advantage of enhancing the resistance to impact, ductility, and eliminates its brittleness.

Different guidelines have suggested different methods such as projectile impact test and falling-weight test to evaluate the impact strength of concrete. In particular, the falling-weight test method is the simple one suggested by the ACI Committee 544 [2]. The extensive experimental studies were conducted to evaluate the impact strength of fibrous concrete using falling weight test, and large scatter in results were observed in previous studies [1-13]. The reason for massive scattering in falling weight test outcomes can be ascribed as follows: the crack initiation and damage are visually monitoring; the impact load applied was a target one location of concrete which might be hit either a region of coarse aggregate or on a weak region of mortar matrix; concrete is non- 
homogeneous material; weight drops from a particular height, it is hard to manage due to the manual work action. Consequently, the best choice to deal with scattered results by two-parameter distribution of Weibull.In this study, the experimental impact test results were collected from earlier literature (Table 1) and analysed using the energy pattern factor method. Twenty equations were proposed to evaluate the shape parameter, and four levels of validation were employed to verify the accuracy of equations.

\section{DISTRIBUTION OF WEIBULL}

The distribution of Weibull was used for the first time by Wallodi Weibull [14]. The field of application of this distribution is immense and covers almost all areas of research. Hence, the estimation of the parameter is considered a key theme because of the precision of possibility and reliability analysis. Power density method, wasp method, justus moment method, moment method, modified maximum likelihood method, alternative maximum likelihood method, maximum likelihood method, and graphic method. These are widely deployed methods that have their beneficial and negative impacts.

\section{SHAPE PARAMETER ESTIMATION}

A energy pattern factor method was used to propose new equations through curve fitting technique. Impact resistance of concrete and its distribution are major criteria for design of structure to resist impact loading. The ratio of mean of cubic impact strength (number of impacts) and the cube of mean impact strength is described by energy pattern factor (epf) which can be stated by the Eq (1) as follows.

$$
E_{p f}=\frac{\overline{N^{3}}}{\left(N_{m}\right)^{3}}
$$

The shape parameter can be determined based on the Epf value is expressed by the Eq(2) as follows.

$$
k=1+\frac{3.69}{\left(E_{p f}\right)^{2}}
$$

Where,

$\overline{N^{3}}=$ Mean of impact strengthcubes, $\left(N_{m}\right)^{3}=$ Cube of mean impact strength, $\mathrm{k}=$ Shape parameter and epf $=$ Energy pattern factor.

The graph is plotted between the epf is on $\mathrm{x}$ axis and $\mathrm{k}$ (shape parameter) is on $\mathrm{y}$ axis and the curve fitting software was used to propose the 20 equations. The detailed procedure for proposing and four level validations are clearly illustrated in Figure 1.

Figure 2 illustrates the sample curve fitting using epf and $\mathrm{k}$ values. The following twenty equation were selected based on the regression coefficient value greater than 0.9 and this process is executes with the help of curve fitting software tool. 
A new energy pattern factor method to estimate Weibull shape parameter for impact resistance of concrete

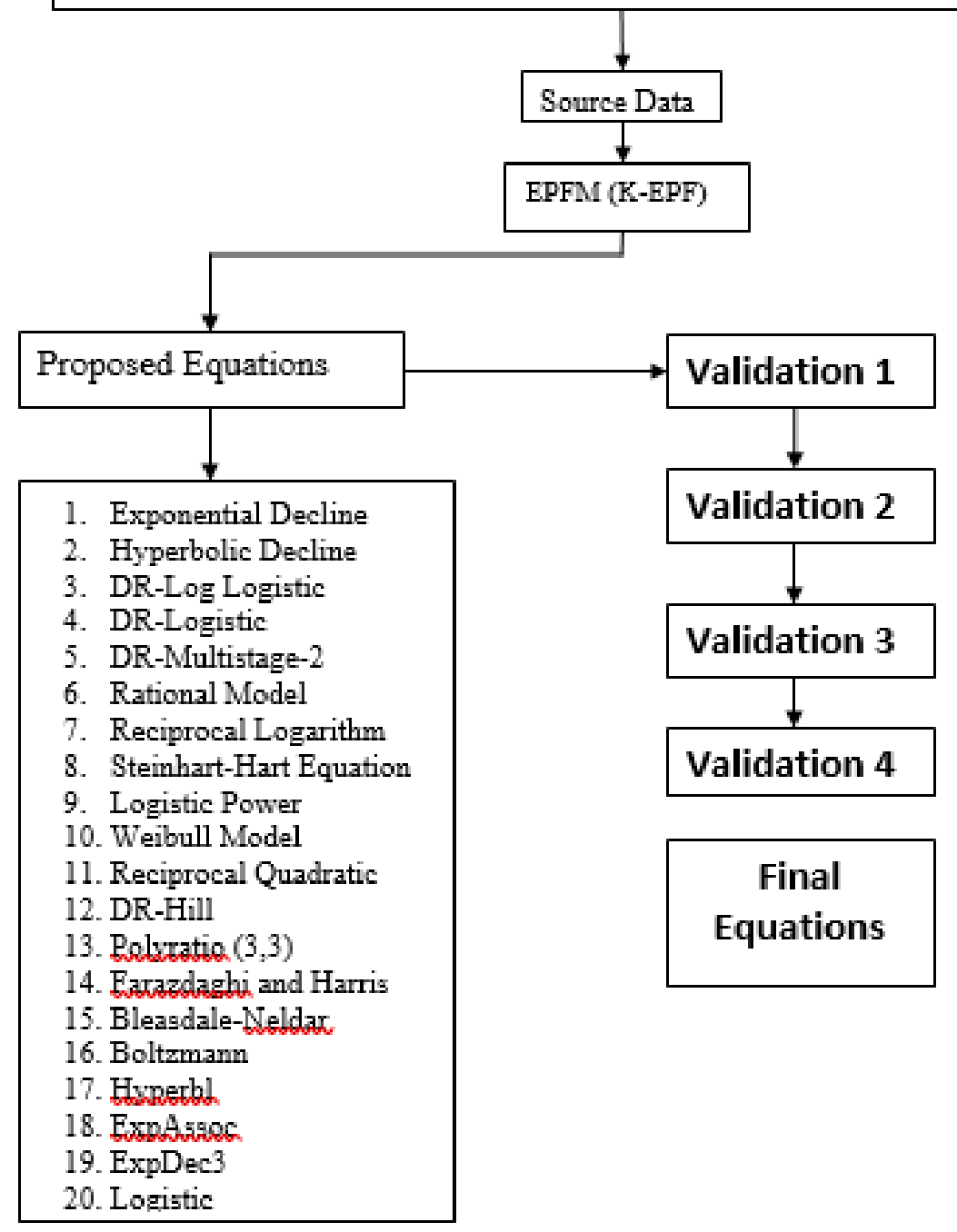

Figure 1: Methodology of the proposed equation

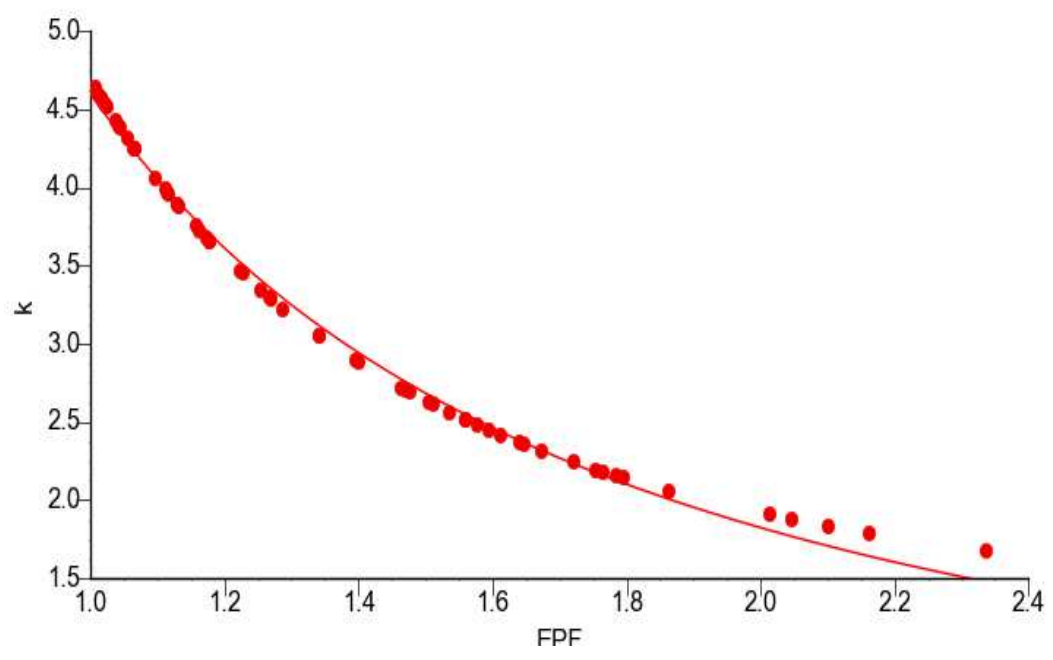

Figure 2: Hyperbolic decline curve fitting. 
Table 1: Source data collected from earlier literatures

\begin{tabular}{|c|c|c|c|c|c|c|c|}
\hline $\begin{array}{c}\text { Referenc } \\
\text { e }\end{array}$ & $\begin{array}{l}\text { Type of } \\
\text { concrete }\end{array}$ & Mix Id & $\begin{array}{l}\text { Type of } \\
\text { fibre }\end{array}$ & $\begin{array}{l}\text { Fibre } \\
\text { dosage }\end{array}$ & $\begin{array}{c}\text { Number } \\
\text { of } \\
\text { specimens } \\
\text { per mix }\end{array}$ & $\begin{array}{c}\text { Mean } \\
\text { N2 }\end{array}$ & SD \\
\hline [1] & $\begin{array}{c}\text { Fibre } \\
\text { Reinforced } \\
\text { Concrete } \\
\end{array}$ & FRC,PC & SF & $0.5,0 \%$ & 15,16 & 139,77 & 64,36 \\
\hline$[2]$ & $\begin{array}{c}\text { Fibre } \\
\text { Reinforced } \\
\text { Concrete } \\
\end{array}$ & SPHRC,SFRC & $\begin{array}{l}\mathrm{CF}+\mathrm{PPF}, \\
\mathrm{CF}\end{array}$ & $0.5+0.1,0.5 \%$ & 48 & 356,330 & $\begin{array}{l}146 \\
172\end{array}$ \\
\hline [4] & $\begin{array}{c}\text { High } \\
\text { Strength } \\
\text { Concrete } \\
\end{array}$ & HSFRC,HSC & SF & $1 \%, 0 \%$ & 48 & 1896,454 & $\begin{array}{c}802,18 \\
5\end{array}$ \\
\hline$[5]$ & $\begin{array}{c}\text { Fibre } \\
\text { Reinforced } \\
\text { Concrete } \\
\end{array}$ & $\begin{array}{c}\text { PPFRC (BATCH } \\
\text { 1,BATCH 2) }\end{array}$ & PPF & $3 \mathrm{~kg} / \mathrm{m} 3$ & 20 & 84,76 & 44,37 \\
\hline [6] & $\begin{array}{c}\text { High } \\
\text { Performance } \\
\text { Concrete }\end{array}$ & $\begin{array}{l}\text { NC,SFRC20,SFR } \\
\text { C35,RC,RC+SFR } \\
\text { C20,RC+SFRC35 }\end{array}$ & SF & $\begin{array}{c}0,20,35,0,20,35 \\
\mathrm{~kg} / \mathrm{m} 3\end{array}$ & 6 & $\begin{array}{c}15,210,407,3 \\
50,1196,123 \\
2\end{array}$ & $\begin{array}{c}7,142,1 \\
36,128, \\
322,28 \\
0\end{array}$ \\
\hline [7] & $\begin{array}{c}\text { Fibre } \\
\text { Reinforced } \\
\text { Concrete }\end{array}$ & $\begin{array}{c}\text { PC,CFRC,PFRC,S } \\
\text { FRC }\end{array}$ & $\begin{array}{l}\mathrm{CF}^{*}, \mathrm{PF}, \\
\mathrm{SF}\end{array}$ & $\begin{array}{c}0 \%, 0.15 \% \\
0.15 \%, 0.5 \%\end{array}$ & 32 & $\begin{array}{c}48,118,71,22 \\
8\end{array}$ & $\begin{array}{c}28,53,3 \\
6,90\end{array}$ \\
\hline [8] & $\begin{array}{c}\text { Self- } \\
\text { Compacting } \\
\text { Concrete } \\
\text { with } \\
\text { recycled } \\
\text { fibres }\end{array}$ & $\begin{array}{c}\text { CFRP-0.25,CFRP- } \\
0.75, \text { CFRP- } 1.25\end{array}$ & $\mathrm{CCF}$ & $\begin{array}{c}0.25 \%, 0.75 \%, 1.25 \\
\%\end{array}$ & 40 & $51,77,104$ & $\begin{array}{c}18,29,4 \\
1\end{array}$ \\
\hline [9] & $\begin{array}{c}\text { High } \\
\text { Performance } \\
\text { Self- } \\
\text { Compacting } \\
\text { Concrete } \\
\end{array}$ & $\begin{array}{c}\text { NC,PP4,PP6,SF20 } \\
\text {,SF35,RC,RC+PP } \\
\text { 4,RC+PP6,RC+SF } \\
\text { 20,RC+SF35 }\end{array}$ & PPF,SF & $\begin{array}{c}0,4,6,20,35,0,4,6 \\
20 \\
35 \mathrm{~kg} / \mathrm{m} 3\end{array}$ & 6 & $\begin{array}{c}15,33,40,52, \\
55,350,489,7 \\
43,1196,123 \\
2\end{array}$ & $\begin{array}{c}7,7,5,2 \\
7,24,12 \\
8,129,1 \\
93,322 \\
280 \\
\end{array}$ \\
\hline$[10]$ & $\begin{array}{c}\text { Fibre } \\
\text { Reinforced } \\
\text { Concrete }\end{array}$ & $\begin{array}{c}\text { PC,CF-1.5,CF- } \\
\text { 3.0,CF-5.0,HF- } \\
\text { 1.5,HF-3.0,HF-5.0 }\end{array}$ & $\mathrm{CF}^{* *}, \mathrm{HF}$ & $\begin{array}{c}0 \%, 1.5 \%, 3 \%, 5 \% \\
1.5 \%, 3,5 \%\end{array}$ & 15 & $\begin{array}{c}84,312,737,1 \\
209,424,918, \\
1378\end{array}$ & $\begin{array}{c}25,86,1 \\
13,151 \\
64,78,1 \\
22\end{array}$ \\
\hline [11] & $\begin{array}{c}\text { Fibre } \\
\text { Reinforced } \\
\text { Concrete }\end{array}$ & $\begin{array}{c}\text { PC,FRC,TSC,TSF } \\
\text { RC,SIFCON,LFR } \\
\text { C }\end{array}$ & SF & $\begin{array}{c}0 \%, 1 \%, 1.5 \%, 0 \% \\
4 \%, 5 \%, 8 \%, 10 \%, 1 \\
.33 \%, 1.66 \%\end{array}$ & 6 & $\begin{array}{c}36,305,374,5 \\
8,1175,1358, \\
1858,2074,1 \\
261,1394\end{array}$ & $\begin{array}{c}9,65,60 \\
, 15,153 \\
, 94,94, \\
164,12 \\
4,109 \\
\end{array}$ \\
\hline [12] & $\begin{array}{c}\text { Geo } \\
\text { polymer } \\
\text { Concrete }\end{array}$ & HFGC & $\begin{array}{c}\text { SF,PF,G } \\
\text { F }\end{array}$ & $\begin{array}{c}0 \%, 1.6 \%, 0.3 \%, 0 \\
3 \%, 1.6 \%, 1.6 \%, 0 \\
6 \%, 1.6 \%\end{array}$ & 5 & $\begin{array}{c}14,101,32,35 \\
, 135,110,45 \\
148\end{array}$ & $\begin{array}{c}5,20,10 \\
, 12,52 \\
25,20,2 \\
2 \\
\end{array}$ \\
\hline [13] & ACI-544R & $\mathrm{PC}$ & - & - & 15 & $\begin{array}{c}358,417,693 \\
1334,107,17 \\
1\end{array}$ & $\begin{array}{c}207,18 \\
5,343,7 \\
64,23,8 \\
2\end{array}$ \\
\hline
\end{tabular}

(SF-Steel Fibre, CF-Collated Fibres, PPF-Polypropylene Fibres, CF*-Cellulose Fibres, CCF-Chopped Carbon Fibre, CF**-Crimped Short Fibre, HF-Hooked End Long Fibre, GF-Glass Fiber) 


\section{Exponential Decline}

$y=q_{0} e^{-x / a}$

$\mathrm{q}_{0}=11.7794263065183 ; \mathrm{a}=1.03173478704351$

\section{Hyperbolic Decline}

$y=q_{0}\left[1+\frac{b x}{a}\right]^{-1 / b}$

$\mathrm{q}_{0}=285.209479586028 ; \mathrm{a}=0.0387091933819022 ; \mathrm{b}=0.721310403127739$

\section{DR-Log Logistic}

$y=\gamma+\frac{1-\gamma}{1+e^{-\alpha-\beta \ln x}}$

$\Upsilon=1046.21047925558 ; \alpha=4.16536446460519 ; \beta=1.51175291645453$

\section{DR-Logistic}

$y=\gamma+\frac{1-\gamma}{1+e^{-\alpha-\beta x}}$

$\Upsilon=1046.21047925558 ; \alpha=4.16536446460519 ; \beta=1.51175291645453$

\section{DR-Multistage-2}

$y=\gamma+(1-\gamma)\left[1-e^{-\beta_{1} x-\beta_{2} x^{2}}\right]$

$\Upsilon=39.0687851410823 ; \beta_{1}=2.82119647274143 ; \beta_{2}=-0.480965193404492$

\section{Reciprocal Logarithm}

$y=\frac{1}{a+b \ln (x)}$

$\mathrm{a}=0.208928012277249 ; \mathrm{b}=0.420634288415268$

\section{Rational Model}

$y=\frac{a+b x}{1+c x+d x^{2}}$

$a=-3612794.14348881 ; b=6971795.34423956 ; c=-1294917.23940168 ; d=2007670.56313117$

\section{Steinhart-Hart Equation}

$y=\frac{1}{A+B \ln (x)+C \ln (x)^{3}}$

$\mathrm{A}=0.21137252287346 ; \mathrm{B}=0.384377691843997 ; \mathrm{C}=0.135183284699429$

\section{Logistic Power}

$y=\frac{a}{\left[1+\left(\frac{x}{b}\right)^{c}\right]}$

$\mathrm{a}=150.04771609523 ; \mathrm{b}=0.0792935227794693 ; \mathrm{c}=1.36259606808846$ 


\section{Weibull Model}

$y=a-b e^{-c x^{d}}$

$\mathrm{a}=28.7913004950343 ; \mathrm{b}=27.7241189630685 ; \mathrm{c}=0.139856635504285 ; \mathrm{d}=-2.15645880997987$

\section{Reciprocal Quadratic}

$y=\frac{1}{\left(a+b x+c x^{2}\right)}$

$\mathrm{a}=-0.183939802325021 ; \mathrm{b}=0.440412220423591 ; \mathrm{c}=-0.0439273875912001$

\section{DR-Hill}

$y=\alpha+\frac{\theta \mathrm{x}^{\eta}}{\kappa^{\eta}+x^{\eta}}$

$\alpha=1.05239285240186 ; \theta=67.6007709569144 ; \eta=-2.12235537735702 ; k=0.258907594087375$

\section{Polyratio $(3,3)$}

$y=\frac{A+B x+C x^{2}+D x^{3}}{1+E x+F x^{2}+G x^{3}}$

$\mathrm{A}=21.4955 ; \mathrm{B}=12.42382 ; \mathrm{C}=6.35135 ; \mathrm{D}=1.52057 ; \mathrm{E}=-3.53658 ; \mathrm{F}=10.46434 ; \mathrm{G}=0.98316$

\section{Farazdaghi and Harris}

$y=\frac{1}{\left(A+B x^{c}\right)}$

$\mathrm{A}=-0.36515 ; \mathrm{B}=0.57751 ; \mathrm{C}=0.61974$

\section{Bleasdale-Nelder}

$y=(A+B x)^{-1 / c}$

$\mathrm{A}=-0.16145 ; \mathrm{B}=0.29847 ; \mathrm{C}=1.28258$

\section{Boltzmann}

$y=\frac{A_{1}+A_{2}}{1+e^{\left(x-x_{0}\right) / d x}}+A_{2}$

$A_{1}=807.766 ; A_{2}=1.56305 ; x_{0}=-1.59948 ; d x=0.4676$

\section{Hyperbl}

$y=\frac{p_{1} x}{p_{2}+x}$

$\mathrm{p}_{1}=1.35816 ; \mathrm{p}_{2}=-0.72318$

18. ExpAssoc

$y=y_{0}+A_{1}\left(1-e^{-x / t_{1}}\right)+A_{2}\left(1-e^{-x / t_{2}}\right)$

$\mathrm{y}_{0}=48.57357 ; \mathrm{A}_{1}=-4.20023 ; \mathrm{t}_{1}=1.30301 ; \mathrm{A}_{2}=-43.42683 ; \mathrm{t}_{2}=0.3135$ 


\section{ExpDec3}

$y=y_{0}+A_{1} e^{-x / t_{1}}+A_{2} e^{-x / t_{2}}+A_{3} e^{-x / t_{3}}$

$\mathrm{y}_{0}=1.56116 ; \mathrm{A}_{1}=8.69269 ; \mathrm{t}_{1}=0.46923 ; \mathrm{A}_{2}=8.69269 ; \mathrm{t}_{2}=0.46923 ; \mathrm{A}_{3}=8.69269 ; \mathrm{t}_{3}=0.46923$

\section{Logistic}

$y=\frac{A_{1}-A_{2}}{1+\left(x / x_{0}\right)^{p}}+A_{2}$

$\mathrm{A}_{1}=74.92525 ; \mathrm{A}_{2}=1.04813 ; \mathrm{x}_{0}=0.24618 ; \mathrm{p}=2.11185$

\subsection{Validation 1}

From the first validation one, the k values obtained from the Eq (2) is compared with the k values predicted from proposed equations. The equations were selected for the next level of validation based on the percentage difference between the actual and predicted $\mathrm{k}$ values are less than 10 . The equation cleared the first validation is shown in Table 2.

\subsection{Validation 2}

The empirical method of lysen is recommended by the Lysen in 1983 and method of moments were used for the second level of validation of the proposed equations. The shape parameter can be determined using empirical method of lysen and method of moments are expressed in equation (23) and (24). The k values are computed using the aforementioned methods and compared it with the $\mathrm{k}$ values obtained from the equation (2). The equations are processed to the next level of validation, while the percentage error between the actual and predicted values are less than 10. Totally, fourteen equations were processed in level two validation and eight equations were further processed into level three validation.

$$
\begin{aligned}
& k=\left(\frac{\sigma}{\bar{N}}\right)^{-1.086} \\
& k=\left(\frac{0.9874}{\frac{\sigma}{\bar{N}}}\right)^{-1.086}
\end{aligned}
$$

$\sigma=$ Standard Deviation

$\bar{N}=$ Mean impact strength

\subsection{Validation 3}

In order to globalise the proposed equations, the fatigue data's [15-17] were used to calculated the $\mathrm{k}$ values from actual and proposed equations and compared each other. Three equations were cleared the level three validation by comparing the $\mathrm{k}$ values and percentage error was less than $5 \%$.

\begin{tabular}{|c|c|c|c|c|c|c|}
\hline \multirow[b]{2}{*}{$\begin{array}{l}\text { S. } \\
\text { No }\end{array}$} & \multirow[b]{2}{*}{ Equations } & \multirow[b]{2}{*}{$\begin{array}{c}\text { Validation } \\
1\end{array}$} & \multicolumn{2}{|c|}{ Validation 2} & \multirow[b]{2}{*}{$\begin{array}{l}\text { Validation } \\
\quad 3\end{array}$} & \multirow[b]{2}{*}{$\begin{array}{c}\text { Validation } \\
4\end{array}$} \\
\hline & & & $\begin{array}{c}\text { Empirical Method of } \\
\text { Lysen }\end{array}$ & $\begin{array}{l}\text { Method of } \\
\text { Moments }\end{array}$ & & \\
\hline 1. & Exponential Decline & $\checkmark$ & - & - & - & - \\
\hline 2. & Hyperbolic Decline & $\checkmark$ & - & - & - & - \\
\hline 3. & DR-LogLogistic & $\checkmark$ & - & - & - & - \\
\hline 4. & DR-Logistic & $\checkmark$ & - & - & - & - \\
\hline
\end{tabular}

Table 2: Percentage error between the actual and predicted shape parameters 


\begin{tabular}{|c|c|c|c|c|c|c|}
\hline 5. & DR-Multistage-2 & $\checkmark$ & $\checkmark$ & $\checkmark$ & $\checkmark$ & - \\
\hline 6. & $\begin{array}{l}\text { Reciprocal } \\
\text { Logarithm }\end{array}$ & $\checkmark$ & $\checkmark$ & $\checkmark$ & - & - \\
\hline 7. & Rational Model & $\checkmark$ & $\checkmark$ & $\checkmark$ & - & - \\
\hline 8. & $\begin{array}{l}\text { Steinhart-Hart } \\
\text { Equation }\end{array}$ & $\checkmark$ & $\checkmark$ & $\checkmark$ & - & - \\
\hline 9. & Logistic Power & $\checkmark$ & - & - & - & - \\
\hline 10. & Weibull Model & $\checkmark$ & $\checkmark$ & $\checkmark$ & $\checkmark$ & - \\
\hline 11. & $\begin{array}{l}\text { Reciprocal } \\
\text { Quadratic }\end{array}$ & $\checkmark$ & $\checkmark$ & $\checkmark$ & - & - \\
\hline 12. & DR-Hill & $\checkmark$ & $\checkmark$ & $\checkmark$ & $\checkmark$ & $\checkmark$ \\
\hline 13. & Polyratio $(3,3)$ & $\checkmark$ & $\checkmark$ & $\checkmark$ & $\checkmark$ & $\checkmark$ \\
\hline 14. & $\begin{array}{l}\text { Farazdaghi and } \\
\text { Harris }\end{array}$ & $\checkmark$ & $\checkmark$ & $\checkmark$ & - & - \\
\hline 15. & Bleasdale-Nelder & $\checkmark$ & $\checkmark$ & $\checkmark$ & - & - \\
\hline 16. & Boltzmann & $\checkmark$ & $\checkmark$ & $\checkmark$ & $\checkmark$ & - \\
\hline 17. & Hyperbl & $\checkmark$ & - & - & - & - \\
\hline 18. & ExpAssoc & $\checkmark$ & $\checkmark$ & $\checkmark$ & $\checkmark$ & - \\
\hline 19. & ExpDec 3 & $\checkmark$ & $\checkmark$ & $\checkmark$ & $\checkmark$ & - \\
\hline 20. & Logistic & $\checkmark$ & $\checkmark$ & $\checkmark$ & $\checkmark$ & $\checkmark$ \\
\hline
\end{tabular}

Table 3: Results of statistical indicators used for fourth validation

\begin{tabular}{|l|l|l|l|}
\hline \multicolumn{1}{|c|}{ Indicators } & \multicolumn{1}{c|}{ Polyratio (3,3) } & \multicolumn{1}{c|}{ Logistic } & \multicolumn{1}{c|}{ DR-Hill } \\
\hline Root Mean Square Error (RMSE) & 0.000039779 & 0.00134005 & 0.00147406 \\
\hline Mean absolute percentage error (MAPE) \% & 0.001169703 & 0.041052875 & 0.045073718 \\
\hline Mean Absolute Deviation (MAD) & 0.000033540 & 0.001175451 & 0.001293156 \\
\hline Integral Absolute Error (IAE) \% & 0.072521601 & 2.54527822 & 2.794570544 \\
\hline Relative Root Mean Square Error (RRMSE) & 0.001780631 & 0.010541358 & 0.011056553
\end{tabular}

\subsection{Validation 4}

The fourth validation was employed by applying the several statistical indicators. The three equation after the third level of validation were analysed with the statistical indicators as follows [18].

\subsubsection{Root Mean Square Error (RMSE)}

$\operatorname{RMSE}=\sqrt{\frac{1}{\mathrm{n}} \sum_{\mathrm{i}=1}^{\mathrm{n}}\left(\mathrm{E}_{\mathrm{v}}-\mathrm{P}_{\mathrm{v}}\right)^{2}}$

\subsubsection{Mean Absolute Percentage Error (MAPE)}

$\operatorname{MAPE}=\left(\frac{1}{n} \sum_{\mathrm{i}=1}^{\mathrm{n}}\left|\frac{\mathrm{E}_{\mathrm{v}}-\mathrm{P}_{\mathrm{v}}}{\mathrm{P}_{\mathrm{v}}}\right|\right) \times 100 \%$

\subsubsection{Mean Absolute Deviation (MAD)}

$\mathrm{MAD}=\frac{\sum\left|\mathrm{E}_{\mathrm{V}}-\mathrm{P}_{\mathrm{V}}\right|}{\mathrm{n}}$

\subsubsection{Integral Absolute Error (IAE)}

$\mathrm{IAE}=\sum \frac{\left[\left(\mathrm{E}_{\mathrm{V}}-\mathrm{P}_{\mathrm{V}}\right)^{2}\right]^{1 / 2}}{\mathrm{E}_{\mathrm{V}}} \times 100$ 


\subsubsection{Relative Root Mean Square Error (RRMSE)}

RRMSE $=\frac{\sqrt{\frac{1}{n} \sum_{i=1}^{n}\left(E_{v}-P_{v}\right)}}{\frac{1}{n} \sum_{i=1}^{n} E_{v}}$

$\mathrm{E}_{\mathrm{v}}=$ Actual Value; $\mathrm{P}_{\mathrm{v}}=$ Predicted Value

It is clear from the Table 3, all statistical indicators exhibited the values near to zero which indicated the proposed equations are precise and it can be applied to any area of research.

\section{CONCLUSIONS}

In the present study, the energy pattern factor method is developed and signified. Its excellent characteristics are exposed compared to other methods. This new approach was established and proposed the three equations to predict the shape parameter, namely Polyratio (3,3), Logistic, and DR-Hill. Four levels of validation verified the fittingness and accurateness of the three proposed equations. It is necessary to indicate that the superiority of the energy factor method over the other method of Weibull distribution. Therefore, proposed equations to compute the shape parameter can be useful to many areas of research. Besides, this new approach offers not only equivalence in precision but also easy methods for the calculation than traditional methods.

\section{REFERENCES}

1. M.C. Nataraja, N. Dhang, A.P. Gupta. Statistical variations in impact resistance of steel fiber-reinforced concrete subjected to drop weight test. Cement and Concrete Research 29 (1999) 989-995.

2. P.S. Song, J.C. Wu, S. Hwang, B.C. Sheu. Statistical analysis of impact strength and strength reliability of steel-polypropylene hybrid fiber-reinforced concrete. Construction and Building Materials 19 (2005) 1-9.

3. ACI 544.2R-89 (1999). Measurement of properties of fiber reinforced concrete. American Concrete Institute ACI, USA.

4. P.S. Song, J.C. Wu, S. Hwang, B.C. Sheu. Assessment of statistical variations in impact resistance of high strength concrete and high-strength steel fiber-reinforced concrete. Cement and Concrete Research 35 (2005) 393 - 399.

5. Atef Badr, Ashraf F. Ashour, Andrew K. Platten. Statistical variations in impact resistance of polypropylene fibre-reinforced concrete. International Journal of Impact Engineering 32 (2006) 1907-1920.

6. CHEN Xiang-yu, DING Yi-ning, C. Azevedo. Combined effect of steel fibres and steel rebars on impact resistance of high performance concrete. Central South University Press and Springer-Verlag Berlin Heidelberg 2011.

7. Tara Rahmani, Behnam Kiani, Mohammad Shekarchi, Abdollah Safari. Statistical and experimental analysis on the behavior of fiber reinforced concretes subjected to drop weight test. Construction and Building Materials 37 (2012) 360-369.

8. M. Mastali, A. Dalvand. The impact resistance and mechanical properties of self-compacting concrete reinforced with recycled CFRP pieces. Composites Part B 92 (2016) 360 e376.

9. Yining Ding, Dong Li, Yulin Zhang, Cecilia Azevedo. Experimental investigation on the composite effect of steel rebars and macro fibers on the impact behavior of high performance self-compacting concrete. Construction and Building Materials 136 (2017) 495-505.

10. G. Murali, Neha P. Asrani,V. R. Ramkumar, A. Siva \& M. K. Haridharan. Impact Resistance and Strength Reliability of Novel Two-Stage Fibre-Reinforced Concrete. Arabian Journal for Science and Engineering (2019) 44:4477-4490. 
11. T. Abirami, M. Loganaganandan, G. Murali, Roman Fediuk, R. Vickhram Sreekrishna, T. Vignesh,G. Januppriya, K. Karthikeyan. Experimental research on impact response of novel steel fibrous concretes under falling mass impact. Construction and Building Materials 222 (2019) 447-457.

12. Neha P Asrani, G. Murali, K. Parthiban, K. Surya, A. Prakash, K. Rathika, Uma Chandru.A feasibility of enhancing the impact resistance of hybrid fibrous geopolymer composites: Experiments and modelling. Construction and Building Materials 203 (2019) 56-68.

13. Sallal R. Abid, Munther L. Abdul Hussein, Sajjad H. Ali, Ala'a F. Kazem. Suggested modified testing techniques to the ACI 544-R repeated drop-weight impact test. Construction and Building Materials 244 (2020) 118321.

14. Weibull W. A statistical theory of the strength of materials (No. 151).Generalstabens litografiska anstalts förlag; 1939.

15. S. Goel, S.P. Singh, P. Singh, Flexural fatigue strength and failure probability of Self-Compacting Fibre Reinforced Concrete beams. Engineering Structures 40 (2012) 131-140

16. S. Goel, S.P. Singh,Fatigue performance of plain and steel fibre reinforced self-compacting concrete using $S-N$ relationship. Engineering Structures 74 (2014) 65-73

17. Kyu-Seok Yeon, Yoon-Sang Choi, Kwan-Kyu Kim, Jung Heum Yeon, Flexural fatigue life analysis of unsaturated polyestermethyl methacrylate polymer concrete. Construction and Building Materials 140 (2017) 336-343.

18. G. Murali, V. Chandana. Weibull reliability analysis of impact resistance on self-compacting concrete reinforced with recycled CFRP pieces. Romanian Journal of Materials 47 (2) (2017) 196 -203. 